\title{
Multifunctional Nanostructures Based on Inorganic Nanoparticles and Oligothiophenes and Their Exploitation for Cellular Studies
}

\author{
Alessandra Quarta, ${ }^{\dagger}$ Riccardo Di Corato, ${ }^{\dagger}$ Liberato Manna, ${ }^{\dagger}$ Simona Argentiere,${ }^{\dagger}$ \\ Roberto Cingolani, ${ }^{\dagger}$ Giovanna Barbarella, ${ }^{\ddagger}$ and Teresa Pellegrino ${ }^{*, \dagger}$ \\ NNL-National Nanotechnology Laboratory of CNR-INFM and IIT Research Unit, Via per \\ Arnesano 16, Km 5, 73100, Lecce, Italy and CNR, Area di Ricerca, ISOF, \\ I-40129 Bologna, Italy
}

Received January 6, 2008; E-mail: teresa.pellegrino@unile.it

\begin{abstract}
The combination of materials that possess different properties (such as, for instance, fluorescence and magnetism) into one single object of nanoscale size represents an attractive challenge for biotechnology, especially for their potential relevance in biomedical applications. We report here the preparation of novel bifunctional conjugates based on the linkage of inorganic nanoparticles to organic oligothiophene fluorophores (OTFs). In comparison to the organic dyes commonly used in bioimaging and more similarly to colloidal quantum dots, OTFs have broad optical absorption spectra, and therefore OTF fluorophores emitting at different colors can be excited with a single excitation source, allowing for easier multiplexing analysis. In this work we show the preparation of OTF-nanoparticle conjugates based on gold and iron oxide nanoparticles and their characterization using different techniques such as gel electrophoresis, photoluminescence spectroscopy, dynamic light scattering, and so on. In addition, by performing an in vitro study on human tumor cells we show that OTF-nanoparticle conjugates emitting at different colors can be used for multiplexing detection. Also, in the case of iron oxide-OTF conjugates, once uptaken by the cells, we show that they preserve both their fluorescent and their magnetic properties.
\end{abstract}

\section{Introduction}

Inorganic nanoparticles (NPs), including noble metal, magnetic, and semiconductor nanocrystals, are emerging as a novel class of probes for medical applications. Superparamagnetic iron oxide NPs, for instance, are currently under investigation because their magnetic properties make them potentially useful as contrast agents for molecular resonance imaging (MRI), ${ }^{1-6}$ magnetic probes capable of being manipulated under an external magnetic field, ${ }^{7-9}$ and hyperthermia agents for cancer treatment. ${ }^{10,11}$ Also, gold NPs have been demonstrated to be useful as

${ }^{\dagger}$ NNL-National Nanotechnology Laboratory of CNR-INFM and IIT Research Unit.

CNR.

(1) Bremer, C.; Ntziachristos, V.; Weissleder, R. Eur. Radiol. 2003, 13 (2), 231-243.

(2) Doubrovin, M.; Serganova, I.; Mayer-Kuckuk, P.; Ponomarev, V.; Blasberg, R. G. Bioconjugate Chem. 2004, 15 (6), 1376-1388.

(3) Okuhata, Y. Adv. Drug Delivery Rev. 1999, 37 (1-3), 121-137.

(4) Roch, A.; Muller, R. N.; Gillis, P. J. Chem. Phys. 1999, 110 (11), 5403-5411.

(5) Wang, Y. X. J.; Hussain, S. M.; Krestin, G. P. Eur. Radiol. 2001, 11 (11), 2319-2331.

(6) Weinmann, H. J.; Ebert, W.; Misselwitz, B.; Schmitt-Willich, H. Eur J. Radiol. 2003, 46 (1), 33-44.

(7) Pankhurst, Q. A.; Connolly, J.; Jones, S. K.; Dobson, J. J. Phys. D: Appl. Phys. 2003, 36 (13), R167-R181.

(8) Reich, D. H.; Tanase, M.; Hultgren, A.; Bauer, L. A.; Chen, C. S.; Meyer, G. J. J. Appl. Phys. 2003, 93 (10), 7275-7280.

(9) Safarik, I.; Safarikova, M. Monatsh. Chem. 2002, 133 (6), 737-759.

(10) Fortin, J. P.; Wilhelm, C.; Servais, J.; Menager, C.; Bacri, J. C.; Gazeau, F. J. Am. Chem. Soc. 2007, 129 (9), 2628-2635. hyperthermal agents as the local temperature around the gold NPs can be increased by laser illumination due to the surface plasmon resonance effect. ${ }^{12,13}$ On the other hand, fluorescent properties stemming from fluorophores of different nature, ranging from the most conventional organic dyes like FITC, Cy3, Cy5, and rhodamine, to inorganic semiconductor NPs, the latter also called quantum dots, have been widely exploited as probes for optical imaging. ${ }^{14-19}$ The combination in one single nanotool of different nanoparticle probes that allow simultaneously for bioseparation, MRI, and/or fluorescent detection that can be used for cellular imaging, biodetection, and biosensing and for cancer treatment opens the door to a broader variety of

(11) Kalambur, V. S.; Han, B.; Hammer, B. E.; Shield, T. W.; Bischof, J. C. Nanotechnology 2005, 16 (8), 1221-1233.

(12) Huang, X.; Jain, P.; El-Sayed, I.; El-Sayed, M. Lasers in Medical Science; 2007.

(13) O’Neal, D. P.; Hirsch, L. R.; Halas, N. J.; Payne, J. D.; West, J. L. Cancer Lett. 2004, 209 (2), 171-176.

(14) Bruchez, M.; Moronne, M.; Gin, P.; Weiss, S.; Alivisatos, A. P. Science 1998, 281 (5385), 2013-2016.

(15) Holmes, K. L.; Lantz, L. M. Protein labeling with fluorescent probes. In Methods in Cell Biology; 2001; Vol. 63, pp 185-204.

(16) Lin, Y.; Weissleder, R.; Tung, C. H. Bioconjugate Chem. 2002, 13 (3), 605-610.

(17) Mishra, A.; Behera, R. K.; Behera, P. K.; Mishra, B. K.; Behera, G. B. Chem. Rev. 2000, 100 (6), 1973-2012.

(18) Sun, W. C.; Gee, K. R.; Klaubert, D. H.; Haugland, R. P. J. Org. Chem. 1997, 62 (19), 6469-6475.

(19) Wang, F.; Tan, W. B.; Zhang, Y.; Fan, X. P.; Wang, M. Q. Nanotechnology 2006, 17 (1), R1-R13. 
applications. ${ }^{20-29}$ Detection and separation of different molecules in a biological liquid for diagnosis of diseases and more specifically the simultaneous identification of different tumor cell populations for investigation of cancer at a premature phase of its development are, for instance, key issues that can be better addressed by magnetic-fluorescent nanoprobes. ${ }^{30-34}$ As a further example, an in vivo application that can benefit from the use of magnetic-fluorescent conjugates is certainly the dual modal imaging of an organ or a tissue of a clinical patient. ${ }^{35-39}$ Here, the magnetic portion would allow for MRI imaging to capture the patient condition in a presurgical phase, while the fluorescent portion would act as a fluorescent guide during the surgery as it could offer a higher resolution than the MRI tool for the resection of the tumor area. Only recently, fluorescentgold-based conjugates have been exploited as fluorescent probes for detecting biomolecules ${ }^{40}$ small analytes, ${ }^{41}$ and ions ${ }^{42}$ in in vitro assays. ${ }^{43}$ In these tests, the species that has to be determined modulates the fluorescence energy transfer between the gold nanoparticles and the proximal dyes allowing for their quantitave detections. While no studies of such gold-fluorescentbased conjugate for in vivo applications have been reported so far, such conjugates might be seen as bioprobes in which the fluorescent portion of the conjugate would act as a imaging tool for detecting a tumor mass (due to the fluorescent portion with properly tailored spectral features), while the gold domain would act as a therapeutic agent for performing hyperthermia treatment. ${ }^{13,44-46}$ Such important goals drive part of the research

(20) Zebli, B.; Susha, A. S.; Sukhorukov, G. B.; Rogach, A. L.; Parak, W. J. Langmuir 2005, 21 (10), 4262-4265.

(21) Lu, C. W.; Hung, Y.; Hsiao, J. K.; Yao, M.; Chung, T. H.; Lin, Y. S.; Wu, S. H.; Hsu, S. C.; Liu, H. M.; Mou, C. Y.; Yang, C. S.; Huang, D. M.; Chen, Y. C. Nano Lett. 2007, 7 (1), 149-154.

(22) Lin, Y. S.; Wu, S. H.; Hung, Y.; Chou, Y. H.; Chang, C.; Lin, M. L.; Tsai, C. P.; Mou, C. Y. Chem. Mater. 2006, 18 (22), 5170-5172.

(23) Holzapfel, V.; Lorenz, M.; Weiss, C. K.; Schrezenmeier, H.; Landfester, K.; Mailander, V. J. Phys.: Condens. Matter 2006, 18 (38), S2581-S2594.

(24) Guo, J.; Yang, W. L.; Wang, C. C.; He, J.; Chen, J. Y. Chem. Mater. 2006, 18 (23), 5554-5562.

(25) Corr, S. A.; O'Byrne, A.; Gun'ko, Y. K.; Ghosh, S.; Brougham, D. F.; Mitchell, S.; Volkov, Y.; Prina-Mello, A. Chem. Commun. 2006, (43), 4474-4476.

(26) Bertorelle, F.; Wilhelm, C.; Roger, J.; Gazeau, F.; Menager, C.; Cabuil, V. Langmuir 2006, 22 (12), 5385-5391.

(27) Quarta, A.; Di Corato, R.; Manna, L.; Ragusa, A.; Pellegrino, T. IEEE Trans. NanoBiosci. 2007, 6 (4), 298.

(28) Selvan, S. T.; Patra, P. K.; Ang, C. Y.; Ying, J. Y. Angew. Chem., Int. Ed. 2007, 46 (14), 2448-2452.

(29) Corr, S. A.; Rakovich, Y. P.; Gun'ko, Y. K. Nanoscale Res. Lett. 2008, 3 (3), 87-104.

(30) Chu, M. Q.; Song, X.; Cheng, D.; Liu, S. P.; Zhu, J. Nanotechnology 2006, 17 (13), 3268-3273.

(31) Gupta, A. K.; Gupta, M. Biomaterials 2005, 26 (18), 3995-4021.

(32) Wang, D. S.; He, J. B.; Rosenzweig, N.; Rosenzweig, Z. Nano Lett. 2004, 4 (3), 409-413.

(33) Wang, G. P.; Song, E. Q.; Xie, H. Y.; Zhang, Z. L.; Tian, Z. Q.; Zuo, C.; Pang, D. W.; Wu, D. C.; Shi, Y. B. Chem. Commun. 2005, (34), $4276-4278$.

(34) Yoon, T. J.; Yu, K. N.; Kim, E.; Kim, J. S.; Kim, B. G.; Yun, S. H.; Sohn, B. H.; Cho, M. H.; Lee, J. K.; Park, S. B. Small 2006, 2 (2), 209-215.

(35) Frangioni, J. V. Curr. Opin. Chemical. Biol. 2003, 7 (5), 626-634.

(36) Kircher, M. F.; Mahmood, U.; King, R. S.; Weissleder, R.; Josephson, L. Cancer Res. 2003, 63 (23), 8122-8125.

(37) Sevick-Muraca, E. M.; Houston, J. P.; Gurfinkel, M. Curr. Opin. Chem. Biol. 2002, 6 (5), 642-650.

(38) Weissleder, R. Nat. Biotechnol. 2001, 19 (4), 316-317.

(39) Kamaly, N.; Kalber, T.; Ahmad, A.; Oliver, M. H.; So, P. W.; Herlihy, A. H.; Bell, J. D.; Jorgensen, M. R.; Miller, A. D. Bioconjugate Chem. 2008, 19 (1), 118-129.

(40) Kim, J. H.; Estabrook, R. A.; Braun, G.; Lee, B. R.; Reich, N. O. Chem. Commun. 2007, (42), 4342-4344. in nanobioscience toward the development of novel types of multifunctional nanoparticle-based conjugates to the study of their interaction with living cells and exploitation in biomedical applications. The suitability for the applications mentioned above is strongly related to the final size of the nanostructures employed, maintenance of the intrinsic properties of the different portions that form the nanostructures, their surface biochemistry, and not least importantly their biocompatibility. All these features are tightly associated to the approach chosen to prepare multimodal nanostructures and selection of the specific type of fluorescent, magnetic, and noble metal domains. So far, preparation of nanometer-sized magnetic-fluorescent conjugates has exploited different routes, which can be grouped in the following three main categories: (i) encapsulation of both magnetic and fluorescent or metallic and fluorescent probes within a shell of either an organic or an inorganic material; ${ }^{21,22,30,33,34,39,42,47-50}$ (ii) linkage between the two types of probes by means of crosslinker molecules; ${ }^{26,32,36,40,41,51-53}$ (iii) direct synthesis of a bifunctional nanostructure formed by two inorganic domains attached together without the need of cross-linker molecules. ${ }^{54-57}$

We report here the preparation of a new type of hybrid bifunctional nanostructure based on colloidal NPs conjugated to fluorescent oligothiophenes (OTFs) by means of polyethylene glycol (PEG) molecules acting as spacers. OTFs are characterized by tunable emission spectra that depend on structural features, such as the number of the thiophene rings and the substituents grafted to the aromatic backbone. ${ }^{58,59}$ They are characterized by a generally good photochemical stability and large Stokes shifts, are only a few nanometers long, and in

(41) Shang, L.; Qin, C. J.; Wang, T.; Wang, M.; Wang, L. X.; Dong, S. J. J. Phys. Chem. C 2007, 111 (36), 13414-13417.

(42) Chen, J.; Zheng, A.; Chen, A.; Gao, Y.; He, C.; Kai, X.; Wu, G.; Chen, Y. Anal. Chim. Acta 2007, 599 (1), 134-142.

(43) Wang, W.; Xiong, T.; Cui, H. Langmuir 2008, 24 (6), 2826-2833.

(44) Tong, L.; Zhao, Y.; Huff, T. B.; Hansen, M. N.; Wei, A.; Cheng, J. X. Adv. Mater. 2007, 19 (20), 3136-+

(45) Everts, M.; Saini, V.; Leddon, J. L.; Kok, R. J.; Stoff-Khalili, M.; Preuss, M. A.; Millican, C. L.; Perkins, G.; Brown, J. M.; Bagaria, H.; Nikles, D. E.; Johnson, D. T.; Zharov, V. P.; Curiel, D. T. Nano Lett. 2006, 6 (4), 587-591.

(46) Visaria, R. K.; Griffin, R. J.; Williams, B. W.; Ebbini, E. S.; Paciotti, G. F.; Song, C. W.; Bischof, J. C. Mol. Cancer Ther. 2006, 5 (4), $1014-1020$.

(47) Hong, X.; Li, J.; Wang, M. J.; Xu, J. J.; Guo, W.; Li, J. H.; Bai, Y. B.; Li, T. J. Chem. Mater. 2004, 16 (21), 4022-4027.

(48) Salgueirino-Maceira, V.; Correa-Duarte, M. A.; Spasova, M.; LizMarzan, L. M.; Farle, M. Adv. Funct. Mater. 2006, 16 (4), 509-514.

(49) Sathe, T. R.; Agrawal, A.; Nie, S. M. Anal. Chem. 2006, 78 (16), $5627-5632$

(50) Yi, D. K.; Selvan, S. T.; Lee, S. S.; Papaefthymiou, G. C.; Kundaliya, D.; Ying, J. Y. J. Am. Chem. Soc. 2005, 127 (14), 4990-4991.

(51) Josephson, L.; Kircher, M. F.; Mahmood, U.; Tang, Y.; Weissleder, R. Bioconjugate Chem. 2002, 13 (3), 554-560.

(52) Lee, J. H.; Jun, Y. W.; Yeon, S. I.; Shin, J. S.; Cheon, J. Angew. Chem. Int. Ed. 2006, 45 (48), 8160-8162.

(53) Veiseh, O.; Sun, C.; Gunn, J.; Kohler, N.; Gabikian, P.; Lee, D.; Bhattarai, N.; Ellenbogen, R.; Sze, R.; Hallahan, A.; Olson, J.; Zhang, M. Q. Nano Lett. 2005, 5 (6), 1003-1008.

(54) Du, G. H.; Liu, Z. L.; Lu, Q. H.; Xia, X.; Jia, L. H.; Yao, K. L.; Chu, Q.; Zhang, S. M. Nanotechnology 2006, 17 (12), 2850-2854.

(55) Gu, H. W.; Zheng, R. K.; Zhang, X. X.; Xu, B. J. Am. Chem. Soc. 2004, 126 (18), 5664-5665.

(56) Kim, H.; Achermann, M.; Balet, L. P.; Hollingsworth, J. A.; Klimov, V. I. J. Am. Chem. Soc. 2005, 127 (2), 544-546.

(57) Seo, W. S.; Lee, J. H.; Sun, X. M.; Suzuki, Y.; Mann, D.; Liu, Z.; Terashima, M.; Yang, P. C.; McConnell, M. V.; Nishimura, D. G.; Dai, H. J. Nat. Mater. 2006, 5 (12), 971-976.

(58) Barbarella, G.; Favaretto, L.; Sotgiu, G.; Antolini, L.; Gigli, G.; Cingolani, R.; Bongini, A. Chem. Mater. 2001, 13 (11), 4112-4122.

(59) Barbarella, G.; Favaretto, L.; Sotgiu, G.; Zambianchi, M.; Fattori, V.; Cocchi, M.; Cacialli, F.; Gigli, G.; Cingolani, R. Adv. Mater. 1999, 11 (16), 1375-1379. 
addition can be prepared with suitable functional groups for linkage to other types of molecules. ${ }^{60-62} \mathrm{In}$ analogy with colloidal quantum dots and differently from the standard organic dyes that are commonly used in detection experiments, OTFs have broad optical absorption spectra. Therefore, different types of OTFs emitting at different colors can be excited with a single excitation source, allowing for easier multiplexing analysis. Furthermore, a new generation of OTFs containing the $\mathrm{N}$ hydroxylsuccinimidyl ester group (NHS-OTF) has been synthesized recently. ${ }^{63}$ The presence of this carboxylic-activated moiety makes the linkage procedure to amino-modified NPs rather simple and reproducible via formation of amide bonds. ${ }^{64}$ To demonstrate the versatility of this approach we conjugated different types of OTFs emitting at various colors to colloidal NPs. The procedure that we report here offers the further advantage of being easily applicable to the preparation of different types of OTF-NP conjugates. This is possible due to the general approach used for transferring in water the NPs and that delivers NPs characterized by different types of inorganic core materials but always with the same type of surface chemistry ${ }^{65}$ As a proof of principle, we report here OTF-NP conjugates based on both polymer-coated iron oxide and gold NPs. We fully characterized such conjugates and studied their interaction with tumor cells. We have shown their use as multiplexing probes for fluorescent detection and have carried out a cytotoxicity study on such conjugates that aimed at assessing their biocompatibility. Furthermore, in the case of OTF-iron oxide conjugates we also proved the viability of such conjugates, once uptaken by the cells, in cell accumulation by means of magnetic attraction.

\section{Experimental Section}

2.1. Chemicals. All chemicals were used as received. Sodium tetraborate decahydrate (S9640), boric acid (B1934), N-(3-dimethylaminopropyl)- $N^{\prime}$-ethylcarbodiimide hydrochloride (EDC, E7750), Tris-borate-EDTA buffer (T3913), 3-(4,5-dimethyl-2-thiazolyl)-2,5diphenyl-2H-tetrazolium bromide (MTT salt 135038), as well as all the disposable materials and products needed for cell culture were purchased from Sigma-Aldrich. Diamine-PEG 897 (06703), diamine-PEG 2000 (14501), and diamine-PEG 3000 (14502) were purchased from Fluka. Diamine-PEG 3400 (2V2V0F22) was purchased from Nektar. Diamine-PEG 6000 (11-6000-2) was purchased from Rapp-polymer. Agarose (D-1 low EEO, C1349) was purchased from Eppendorf. All solvents used were of analytical grade and purchased from Sigma-Aldrich. Ultrapure-grade water having a conductivity of $18.2 \mu \Omega$ was used in all experiments.

2.2. NP Synthesis and Water Solubilization. Nearly sphericalshaped and monodispersed colloidal $\gamma-\mathrm{Fe}_{2} \mathrm{O}_{3}$ and Au NPs with average diameters of 8 and $6 \mathrm{~nm}$, respectively, were synthesized according to standard procedures ${ }^{66-68}$ (Figure 1s, SI). The concentration of gold NPs was determined by recording their optical adsorption spectra since their optical extinction coefficient is known

(60) Barbarella, G. Chem.-Eur. J. 2002, 8 (22), 5072-5077.

(61) Barbarella, G.; Zambianchi, M.; Pudova, O.; Paladini, V.; Ventola, A.; Cipriani, F.; Gigli, G.; Cingolani, R.; Citro, G. J. Am. Chem. Soc. 2001, 123 (47), 11600-11607.

(62) Sotgiu, G.; Zambianchi, M.; Barbarella, G.; Aruffo, F.; Cipriani, F.; Ventola, A. J. Org. Chem. 2002, 68 (4), 1512-1520.

(63) Barbarella, G.; Zambianchi, M.; Ventola, A.; Fabiano, E.; Della Sala, F.; Gigli, G.; Anni, M.; Bolognesi, A.; Polito, L.; Naldi, M.; Capobianco, M. Bioconjugate Chem. 2006, 17 (1), 58-67.

(64) Hermanson, G. T., Bioconjugate Techniques; Academic Press: San Diego, CA, 1996.

(65) Pellegrino, T.; Manna, L.; Kudera, S.; Liedl, T.; Koktysh, D.; Rogach, A. L.; Keller, S.; Radler, J.; Natile, G.; Parak, W. J. Nano Lett. 2004, 4 (4), 703-707. (for $\mathrm{Au}$ NPs of $6 \mathrm{~nm}$ average diameter this is equal to $8.63 \times 10^{6}$ $\mathrm{cm}^{-1} \mathrm{M}^{-1}$ at $525 \mathrm{~nm}^{69}$ ). In the case of iron oxide NP solutions, since their optical extinction coefficient is not known, the concentration of NPs could be derived by estimating the average number of $\mathrm{Fe}$ atoms per NP (the average NP diameter is assessed via statistical analysis on transmission electron microscopy images of several hundreds of NPs, see below) and determining the total concentration of iron atoms in the solution (via elemental analysis on microwave-digested NP solutions with an ICP-AES spectrophotometer, see also below). As-synthesized NPs are passivated with surfactant molecules, and they are stable in organic solvents. Such NPs could be transferred in water via a polymer-coating procedure that has been reported in a previous work by our group. ${ }^{65}$ Briefly, the NPs were wrapped in an amphiphilic polymer shell made of poly(maleic anhydride alt-1-tetradecene), and this shell was then cross-linked at the surface of the NPs using a triamine. In order to remove the excess unbound polymer the NPs were purified twice via high-performance liquid chromatography (Agilent 1100, instrument) either on a Sephacryl S-300 HR 16/60 column in the case of $\mathrm{Au}$ NPs or on a handmade-packed Sephacryl S-500 HR XK 16/70 column for $\gamma-\mathrm{Fe}_{2} \mathrm{O}_{3} \mathrm{NPs}$, in both cases using as mobile phase a $50 \mathrm{mM}$ phosphate buffer at $\mathrm{pH} 7.0$ containing 150 $\mathrm{mM} \mathrm{NaCl}$ at a flow rate of $0.5 \mathrm{~mL} / \mathrm{min}$.

2.3. Surface Functionalization of NPs with Diamino-PEG. To the NPs delivered from the purification step, the phosphate buffer was exchanged with a $50 \mathrm{mM}$ sodium borate buffer $(\mathrm{pH} 9.0)$ on centrifuge filter tubes (using membranes with pore sizes of 30000 MWCO for $\mathrm{Au}$ NPs and 100000 MWCO for $\gamma-\mathrm{Fe}_{2} \mathrm{O}_{3}$, respectively). The same buffer was used for all of the following reaction steps. Diamino-PEG molecules (NH2-PEG-NH2) of different molecular weights $(897,2000,3000$, and $3400 \mathrm{MW})$ were employed in order to introduce amino moieties at the NP surface (which are needed for the coupling reaction to the NHS-OTF molecules) using EDC as activator of amide bond formation. ${ }^{70}$

By fixing the ratio of PEG molecules added per NP and varying the molar ratio of EDC added to NP solution, it was possible to increase the number of PEG units bound on average to each NP, as previously reported. ${ }^{70}$ The ratio of PEG molecules added per NP was kept constant at 500 as this excess of diamino-PEG molecules was proven to prevent interparticle cross-linking. The conjugation reaction of NPs with diamino-PEG was performed at different EDC/NP ratios, depending on the length of the diaminoPEG molecules and type of NPs. After a reaction time of $3 \mathrm{~h}$ under vigorous stirring at room temperature, the conjugates were run on agarose gel for $1 \mathrm{~h}$ at $100 \mathrm{~V}$ (see details below). Different experimental conditions in the preparation of the conjugates led to mobility profiles that were different from sample to sample. From these "calibration" runs we could therefore set the most appropriate reaction conditions for preparing a specific sample of diamino-PEGfunctionalized NPs with a defined gel retardation band and thus having a specific size (see Figure 2s, SI). On the basis of these preliminary runs, we therefore chose the EDC/NP ratios that are reported in Table 1.

Once the best experimental conditions for the diamino-PEG/NP and EDC/NP ratios were set, the protocol was finalized and consisted of the following steps: (i) mixing together diamino-PEG, EDC, and NPs; (ii) incubation of the mixture for $3 \mathrm{~h}$ under stirring at room temperature; (iii) purification of the reaction mixture on centrifuge tubes (Millipore Amicon tubes) in order to remove excess unreacted PEG molecules.

(66) Brust, M.; Walker, M.; Bethell, D.; Schiffrin, D. J.; Whyman, R. J. Chem. Soc., Chem. Commun. 1994, (7), 801-802.

(67) Sun, S. H.; Zeng, H. J. Am. Chem. Soc. 2002, 124 (28), 8204-8205.

(68) Sun, S. H.; Zeng, H.; Robinson, D. B.; Raoux, S.; Rice, P. M.; Wang, S. X.; Li, G. X. J. Am. Chem. Soc. 2004, 126 (1), 273-279.

(69) Fink, J.; Kiely, C. J.; Bethell, D.; Schiffrin, D. J. Chem. Mater. 1998, 10 (3), 922-926.

(70) Sperling, R. A.; Pellegrino, T.; Li, J. K.; Chang, W. H.; Parak, W. J. Adv. Funct. Mater. 2006, 16 (7), 943-948. 
Table 1. Summary of the EDC/NP Ratios Used To Prepare the Diamino-PEG-Functionalized NPs Reported in This Work

\begin{tabular}{lr}
\hline \multicolumn{1}{c}{ sample name } & EDC/NP \\
\hline Au-PEG897 & 120000 \\
Au-PEG2000 & 16000 \\
Au-PEG3000 & 16000 \\
Au-PEG3400 & 8000 \\
$\mathrm{Au}-\mathrm{PEG} 6000$ & 16000 \\
$\gamma$-Fe & $\mathrm{O}_{3}$-PEG897 \\
$\gamma$ - $\mathrm{Fe}_{2} \mathrm{O}_{3}$-PEG3400 & 4000 \\
\end{tabular}

2.4. Synthesis of Oligothiophene Fluorophores. All OTFs were synthesized according to published procedures. ${ }^{63,71}$ The molecular structure and corresponding spectral position of the maximum of the photoluminescence (PL) of the OTFs most studied in this work are reported in Figure 1a (see also Table 1s). The molecules are OTF1 (5'-methylsulfanyl-[2,2']-bithiophenyl-5-carboxylic acid (2,5dioxopyrrolidin-1-yl) ester; $\left.\lambda_{\mathrm{PL}}(\mathrm{DMSO})=505 \mathrm{~nm}\right)$ and OTF2 $\left(5^{\prime}-\right.$ (4-thienyl-2,1,3-benzothiadiazole)-[2,2']-bithiophenyl-5-carboxylic acid (2,5-dioxopyrrolidin-1-yl) ester; $\left.\lambda_{\mathrm{PL}}(\mathrm{DMSO})=615 \mathrm{~nm}\right)$. The molecular structure and wavelength in the maxima PL emission of all the OTFs that have been studied in this work are reported in Table 1s of the SI together with additional data on preparation of the conjugates and their stability under UV irradiation.

2.5. OTF-NP Conjugation. We performed this reaction in the presence of a ratio of OTF molecules per NP equal to 100. More specifically, a buffer solution of $1 \mu \mathrm{M}$ diamino-PEG-functionalized NPs and a $100 \mu \mathrm{M}$ solution of OTFs dissolved in DMSO were mixed together and left under stirring at room temperature for $96 \mathrm{~h}$. After this time elapsed the samples were washed at least 5 times on centrifuge tubes (15 mL volume, $30000 \mathrm{MWCO})$ and purified twice on NAP-25 columns (Sephadex G-25) in order to remove the unreacted OTF molecules.

2.6. Optical Absorption and Photoluminescence (PL) Measurements. Optical characterization of the starting components and OTF-NP conjugates was carried out using a Varian Cary 300 UV-vis spectrophotometer and a Varian Cary Eclipse fluorescence spectrophotometer. The absorption spectra of the NPs were acquired after synthesis, after following the polymer-coating procedure, and after carrying out surface functionalization with OTFs. The PL spectra of the bare OTF molecules dissolved in DMSO were also compared with those of the OTF-NP conjugates. PL spectra were also recorded on OTF-NP conjugates that had been transferred by dialysis from the aqueous solution to a DMSO solution.

2.7. Characterization by Gel Electrophoresis and Dialysis. Electrophoretic characterization was carried out by running the conjugates through a $2 \%$ agarose gel for a run time of $1 \mathrm{~h}$ at 100 $\mathrm{V}$, after which the gel was observed under both UV light (to locate the OTF signal) and visible light (to locate the NPs). Before the loading step on the gel, to each sample a solution of Orange $\mathrm{G}$ and $30 \%$ glycerol in a gel-loading buffer (corresponding to $20 \%$ of the sample volume) was added. In a series of experiments, the samples, after the gel electrophoretic run, were extracted from the gel and dialyzed (see SI for details).

2.8. Transmission Electron Microscopy (TEM). Low-resolution TEM images were recorded with a Jeol Jem 1011 microscope operating at an accelerating voltage of $100 \mathrm{kV}$. Images were taken on the bare polymer-coated gold and iron oxide NPs and also on the respective NPs functionalized with OTFs. For sample preparation, a drop of a OTF-NP conjugate solution was deposited on carbon-coated grids and the water was allowed to evaporate.

2.9. Compositional Analysis on Nanocrystal Ensembles. An inductively coupled plasma atomic emission spectrometer (Varian Vista AX) was used to investigate the elemental composition of the nanocrystals. The samples were digested in $\mathrm{HCl} / \mathrm{HNO}_{3} 3: 1 \mathrm{(v/}$ v) using a CEM "MARS 5" microwave digester.

(71) Zambianchi, M., B. A.; Ventola, A.; Favaretto, L.; Bettini, C.; Galeotti, M.; Barbarella, G. Bioconjugate Chem. 2007, 18, 1004-1009.
2.10. Dynamic Light Scattering (DLS). DLS measurements were performed on a Zetasizer Nano ZS90 (Malvern) equipped with a $4.0 \mathrm{~mW} \mathrm{He}-\mathrm{Ne}$ laser, operating at $633 \mathrm{~nm}$, and an avalanche photodiode detector. Measurements were carried out at $25{ }^{\circ} \mathrm{C}$ on filtered aqueous solutions of NPs (filtration was performed on a $0.22 \mu \mathrm{m}$ PTFE syringe filter). The average sizes of polymer-coated NPs, the corresponding diamino-PEG-functionalized NPs, and the final OTF-NP conjugates were evaluated and compared with each other. Such comparison provided further characterization of the binding between the diamino-PEG-functionalized NPs and the NHS-OTFs.

2.11. Cell Culture. The human epidermoid carcinoma cells, known as KB cells (ATCC \# CCL-17) were grown continuously as a monolayer at $37{ }^{\circ} \mathrm{C}$ and under $5 \% \mathrm{CO}_{2}$ atmosphere in RPMI1640 medium, supplemented with L-glutamine $(2 \mathrm{~mL})$, penicillin (100 units $/ \mathrm{mL})$, streptomycin $(100 \mu \mathrm{g} / \mathrm{mL})$, and $10 \%$ heatinactivated fetal bovine serum (FBS).

2.12. Cytotoxicity Assay. In order to estimate the toxicity of the conjugates a viability test was carried out using the 3-(4,5dimethyl-2-thiazolyl)-2,5-diphenyl- $2 \mathrm{H}$-tetrazolium bromide assay (MTT assay). ${ }^{72}$ More specifically, $10^{5}$ cells were first seeded in each well of a 12-well plate, and after incubation at $37^{\circ} \mathrm{C}$ for $24 \mathrm{~h}$, the medium was replaced with a fresh medium that contained OTF-NP conjugates at different concentrations (from 1 to $200 \mathrm{nM}$ ). After an additional $24 \mathrm{~h}$ of incubation time at $37{ }^{\circ} \mathrm{C}$, the medium was removed and the cells were washed twice with phosphate buffer (pH 7). Soon after, $1 \mathrm{~mL}$ of a solution containing $1 \mathrm{mg} / \mathrm{mL}$ of MTT dissolved in culture medium was added into each well. After $3 \mathrm{~h}$ of incubation at $37{ }^{\circ} \mathrm{C}$, the MTT, reduced by the mitochondrial reductase of vital cells, formed a dark insoluble product, the formazan. ${ }^{72}$ At this point the medium was collected from each well, centrifuged, and then discarded. The dark pellet was dissolved in DMSO, leading to a violet solution whose absorbance at $570 \mathrm{~nm}$ was determined. The absorbance value can be correlated to the percentage of vital cells, by comparing the data of the doped cells with those of the control cells (i.e., with no conjugates added into medium). In addition, cytotoxicity tests were carried out by loading the cells with the individual components (i.e., either OTFs or bare NPs).

2.13. Magnetic Assay. In order to carry out such assay, the cells were first seeded into a Petri dish $\left(5 \times 10^{4}\right.$ cells per well), after which a solution containing the OTF-NP conjugates (with a concentration equal to $50 \mathrm{nM}$ ) was added to them and the dish was incubated at $37{ }^{\circ} \mathrm{C}$ for $24 \mathrm{~h}$. The medium was then removed, and the adhering cells were washed twice with phosphate buffer. Soon after, the cells were trypsinazed and then seeded in a 6-well plate, underneath which a cylindrical permanent magnet $(\mathrm{NdFeB}$, $0.5 \mathrm{~mm}$ in diameter, corresponding to a total field strength of 1.3 $\mathrm{T}$ ) had been positioned and located in the central part at the bottom of the dish. After incubation for $24 \mathrm{~h}$ at $37^{\circ} \mathrm{C}$, optical images of the adhering cells in the areas above the magnet were acquired, and a statistical analysis was carried out on such images. In order to count only adherent cells, after incubation the medium was exchanged with a fresh medium so as to remove all the cells left in the suspension. For a meaningful statistical analysis, the number of cells seeded in the areas on top of the magnet were counted and compared to the number of adhered cells that had been counted in the control sample (i.e., cells doped with the conjugate but incubated without any magnet underneath the dish). For each sample (well) the experiment was repeated three times, and an average of 30 phase contrast images were recorded on regions on top of the magnet (and similarly for the control experiments).

2.14. Confocal Microscopy Imaging. Confocal microscopy images aimed at assessing cellular uptake, localization, fluorescent signaling of the nanocomposites, and behavior of cells under a magnetic field were recorded on an Olympus FV-1000 microscope equipped with an argon laser source (excitation centered at 488

(72) Slater, T. F.; Sawyer, B.; Strauli, U. Biochim. Biophys. Acta 1963, $77,383$. 


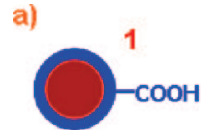

$\mathrm{NH}_{2}-\mathrm{PEG}-\mathrm{NH}_{2}$
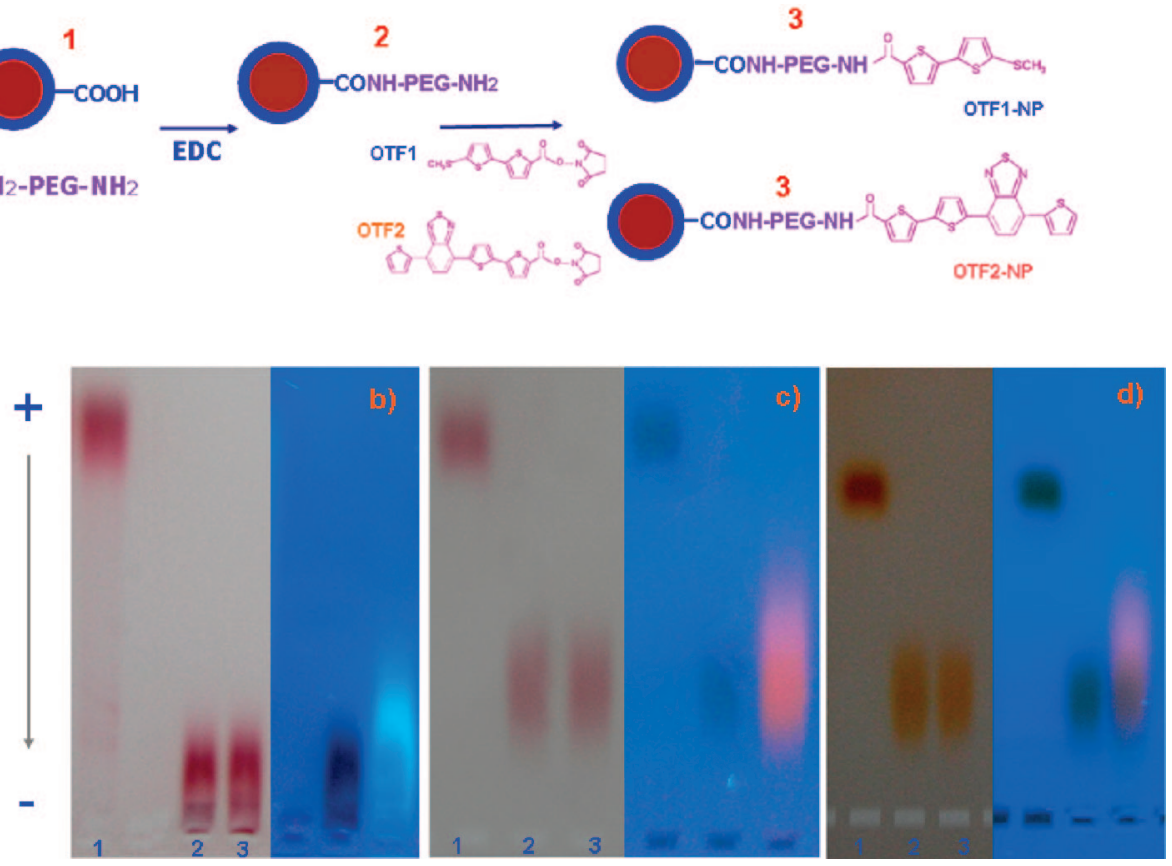

Figure 1. (a) Sketch of the reaction scheme for preparing OTF-NP conjugates. For the reaction, the NPs (brown sphere) were first made soluble in water by wrapping them in an amphiphilic polymer shell based on cross-linked poly anhydride molecules (the blue shell). Hydrolysis of the poly anhydride provides carboxylic groups at the surface of polymer-coated NPs (1), which were reacted with diamino-PEG molecules by means of the coupling agent EDC. Then the diamino-PEG-NPs (2) were reacted with the $N$-hydroxysuccinimidyl ester group of the OTFs to yield the final conjugate by means of an amide bond formation (3). The scheme here refers to linkage of the OTF1 and OTF2 molecules. (b, c, and d) Electrophoresis characterization is reported for the product after each reaction step, namely, polymer-coated (lane 1) NPs, diamino-PEG-functionalized NPs (lane 2), and finally OTF-NP conjugates (lane 3). (b) Gold NPs reacted with diamino-PEG $\left(M_{\mathrm{w}}=3400 \mathrm{~g} / \mathrm{mol}\right)$ and OTF1. (c) Gold NPs reacted with diamino-PEG (MW $\left.=897 \mathrm{~g} / \mathrm{mol}\right)$ and then to the OTF2 molecules. Panel d) refers to $\gamma-\mathrm{Fe}_{2} \mathrm{O}_{3}$ NPs reacted with diamino-PEG $\left(M_{\mathrm{w}}=3400 \mathrm{~g} / \mathrm{mol}\right)$ and then to OTF2. The NPs are visible under white light, whereas the OTFs are detected under UV exposure (excitation wavelength $=365 \mathrm{~nm}$ ). Retardation of the migration is observed for the diamino-PEGfunctionalized NPs as compared to the polymer-coated NPs, while colocalization of bands is observed in the case of the OTF-NP conjugates.

$\mathrm{nm}$ ) with a DM488/405-type dicroic filter and two reading channels having acquisition windows at $527 \pm 10$ and $605 \pm 15 \mathrm{~nm}$, respectively. The $z$-scan images were recorded by focusing on the $x y$ plane of the cells and moving the objective at different heights along the $z$ axis with a step size of $2 \mu \mathrm{m}$.

2.15. Multiplexing Imaging. KB cells at a density of $2 \times 10^{5}$ cells $/ \mathrm{mL} /$ per well were seeded in a 6 -well plate and doped separately with $50 \mathrm{nM}$ concentration of both OTF1-NP and OTF2-NP. After $24 \mathrm{~h}$ at $37^{\circ} \mathrm{C}$, the medium was removed and the cells were washed twice with phosphate buffer, trypsinized, fixed with paraphormaldeide, and then resuspended in $1 \mathrm{~mL}$ of phosphate buffer. An equal amount of cells doped with OTF1-NP and OTF2-NP were mixed together, and a drop of this suspension was loaded onto a glass slide and observed under the confocal microscope using a $405 \mathrm{~nm}$ excitation laser and two different acquisition channels (450 \pm 20 $\mathrm{nm}$ for OTF1 $-\mathrm{NP}$ and $620 \pm 20 \mathrm{~nm}$ for OTF2-NP).

\section{Results and Discussion}

3.1. Preparation of the OTF-NP Conjugates and Their Characterization via Gel Electrophoresis. For the preparation of OTF-NP conjugates we exploited both gold and iron oxide NPs ${ }^{66-68}$ (Figure 1a illustrates the reaction scheme). The surfactant-coated NPs were first transferred from the organic phase to the water phase by embedding them in a polymer shell made of poly(maleicanhydrid unitalicize alt-1-tetradecene) (following a previously published procedure developed by us ${ }^{65}$ ). Hydrolysis of the anhydride groups of the polymer provides the NPs surface of outstretched carboxylic/carboxylate groups, which stabilize the NPs in water by means of electrostatic repulsion. We then bound diamino-PEG molecules to the NP surface by reacting one of the amino groups present on the diamino-PEG molecules with the exposed carboxylic moieties of the outer polymer shell via EDC chemistry. ${ }^{70}$ This procedure introduced amino groups on the surface of the NPs (the unreacted amino groups of the diamino-PEG molecules) and could therefore promote formation of an amidic linkage between NPs and NHS-OTFs according to the reaction NP-NH2 + $\mathrm{NHS}-\mathrm{OTF} \rightarrow \mathrm{NP}-\mathrm{NHC}(\mathrm{O})-\mathrm{OTF}$. The PEG molecules act also as stabilizers as they improve the water stability of NPs and prevent their aggregation under the reaction conditions of the following steps.

Figure $1 b-d$ shows the gel electrophoresis characterization of the product after each reaction step for preparation of the conjugates. On each gel we always ran in parallel the initial polymer-coated NPs, the corresponding diamino-PEG-functionalized NPs, and finally the OTF-NP conjugates. The diaminoPEG-functionalized NPs always showed a retarded migration with respect to the initial polymer-coated NPs, and this was taken as an indication of the successful binding of diaminoPEG molecules to the NP surface. The specific migration of such bands depended both on the conditions under which the diamino-PEG molecules had been reacted with both gold and iron oxides NPs and clearly on their molecular weights, which were either 3400 (Figure 1b) or 2000 (Figure 1c) for the diamino-PEG molecules that had been bound to gold NPs and 3400 for those bound to iron oxide NPs (Figure 1d). The migration bands corresponding to the OTF-NP conjugates always showed a colocalization of the fluorescence signal arising from the OTFs and the optical absorption arising from the inorganic cores (either red-violet for gold $\mathrm{Au}$ or brown for $\gamma-\mathrm{Fe}_{2} \mathrm{O}_{3}$ ) and therefore provided a straightforward indication of the successful binding of the OTFs to the NPs. 
Panels $\mathrm{b}$ and $\mathrm{c}$ of Figure 1 are referred to gold NPs bound to blue-emitting OTFs (named OTF1, $\lambda_{\mathrm{em}}=505 \mathrm{~nm}$ ) and orangeemitting OTFs (named OTF2, $\lambda_{\mathrm{em}}=590 \mathrm{~nm}$ ), respectively, while panel d refers to $\gamma-\mathrm{Fe}_{2} \mathrm{O}_{3}$ NPs bound to orange-emitting OTFs (OTF2).

As a control experiment, bare polymer-coated NPs were mixed with NHS-OTFs. Since these NPs lacked primary amino groups, they should not be able to bind to the NHS-OTF molecules. For this reason, despite the same reaction conditions as reported above for preparing the conjugates being followed in the present case, the final product was washed only partially in order to avoid complete removal of the free OTFs. When this control sample was finally run on the agarose gel, the fluorescent band was not colocalized with the adsorption band of the NPs and as expected its migration on the gel corresponded to that of the free OTF (see, for instance, Figure 5 s of the SI), so no binding occurred. A further gel migration control, which allows us to confirm the binding between the OTFs and the $\mathrm{NP}$, is given by the comparison of the gel migration of the OTF-NP and that of free OTFs in water (for this purpose the OTFs were transferred from DMSO into water, see SI for details). The migration behavior of these free OTFs was different from that of the corresponding OTFs bound to the NPs (it was either faster as OTF1 or slower as OTF2) (see Figure 6s of the SI).

To asses the binding stability of the OTF-NP conjugates we performed a gel extraction and dialysis study, as described in the SI. After applying this procedure and running again the dialyzed OTF-NP conjugates through an agarose gel, colocalization of the UV and visible bands also in this case confirmed the stability of the binding between the NPs and the OTF as the conjugates could stand this procedure while unspecifically bound OTFs would detach from the NPs and pass through the membrane pores (these results are reported in Figure $3 \mathrm{~s}$ of the SI).

3.2. Optical Properties of the Conjugates. The optical adsorption spectra of the conjugates did not show any significant variation with respect to those of the initial NPs (Figure 7s of the SI) as they were mainly dictated by the wide and intense adsorption of the inorganic NP cores in the wavelength range where the OTF molecules also adsorb. The PL profile of the OTF-NP conjugates which were solubilized in water showed, on the other hand, a blue shift with respect to the PL spectrum of the free OTF in DMSO. The entity of this shift depended actually on the type of OTFs considered (being largest for blueemitting OTFs), while it did not seem to depend on the type of NP to which the OTFs were bound (see Figure 2). The shift was about $65 \mathrm{~nm}$ for both OTF1-Au and OTF1- $\gamma-\mathrm{Fe}_{2} \mathrm{O}_{3}$ conjugates and about $18 \mathrm{~nm}$ for both OTF2-Au and OTF2 $-\gamma$ $\mathrm{Fe}_{2} \mathrm{O}_{3}$ conjugates. The shift in the maximum of the emission is likely due to a variation in structural conformation of the OTFs when they are linked to the NP surface with respect to their unbound state in solution.

Interestingly, if the water-soluble OTF1-Au and OTF2-Au conjugates were dialyzed against DMSO, they could be transferred back in DMSO in which they also remained stable and did not undergo any aggregation. We also compared the PL spectra of the OTF-NP conjugates in the aqueous buffer and of the same conjugates in DMSO at the same nanoparticles concentration (i.e., same optical density at $515 \mathrm{~nm}$ ). In the case of the OTF1-Au we did not observe any shift of the PL profile when passing from water to DMSO, whereas an increase of the PL signal was actually recorded (see Figure 3 a). The
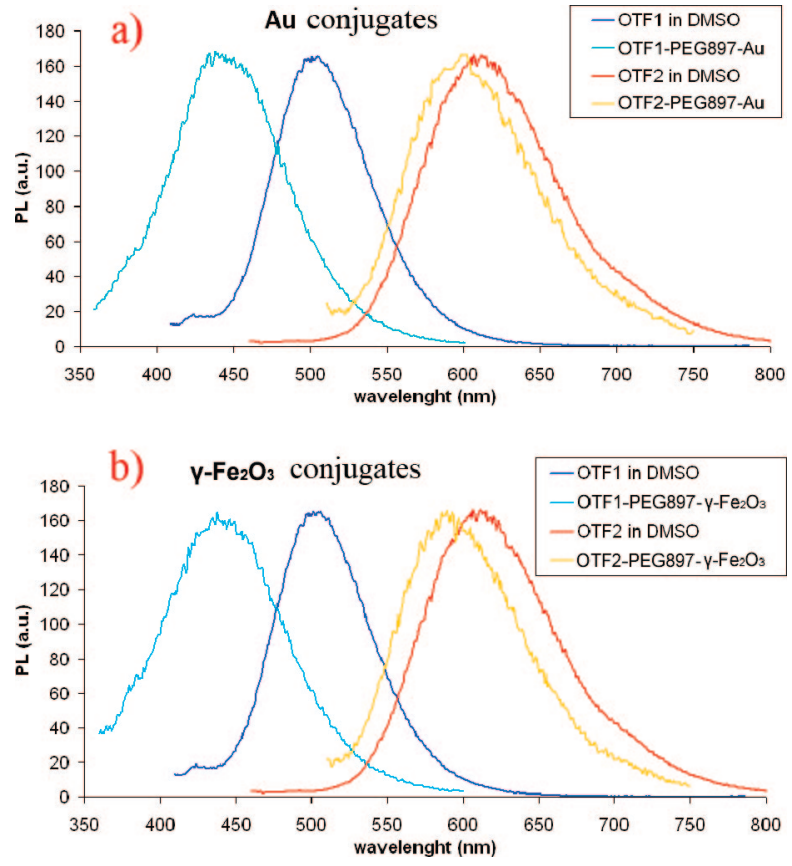

Figure 2. Comparison of emission spectra of OTF1 and OTF2 in DMSO to those of the respective OTF-NP conjugates for (a) gold and (b) iron oxide NPs in water. In all cases the conjugates showed a significant blue shift in the maximum of the emission peak with respect to that of the free OTFs.

maximum of the emission peak for OTF1-Au conjugates both in water and in DMSO was blue shifted as compared to the PL emission from the free OTF1 in DMSO. This feature might indicate that once the OTF1 molecule is linked to the NP it switches to a conformation that is not influenced by the solvent (whether water or DMSO) as no major shift of the PL peak position is observed in the two solvents.

Also, by comparing the PL peak profiles of the conjugates to that of the free OTF1, we could confirm the stability of the linkage between the OTF1 and the NP as again the spectra of the conjugates in water and DMSO are both shifted with respect to that of the free oligothiophene.

The interaction of the conjugate with the solvent can have a significant effect on the emission intensity as an increase of the fluorescent signal is observed when passing from water to DMSO. This behavior is however not general. In the case of the OTF2-Au conjugates, for instance, their transfer from water to DMSO was accompanied by a red shift of about $30 \mathrm{~nm}$ in the PL peak position and an increase in the fluorescent signal was again observed (Figure $3 b$ ). This behavior might be due to a different conformation taken by the OTF2 linked to the NP when the conjugate is dissolved in water with respect to when it is dissolved in DMSO. It is nonetheless remarkable that the PL peak profile of the OTF2-NP conjugate in DMSO is still shifted with respect to that the free OTF in DMSO.

3.3. Dynamic Light Scattering Characterization. The binding of the OTF to the NPs was assessed further by determining the average hydrodynamic diameter of the NPs at each step of the conjugation (polymer-coated NPs, diamino-PEG-functionalized NPs, and OTF-NP conjugates) by means of dynamic light scattering measurements. We found that each of these steps led to an increase in the average hydrodynamic diameter of the NPs. Also, larger increases in NP diameters after the first step of surface anchorage of diamino-PEG molecules could be cor- 

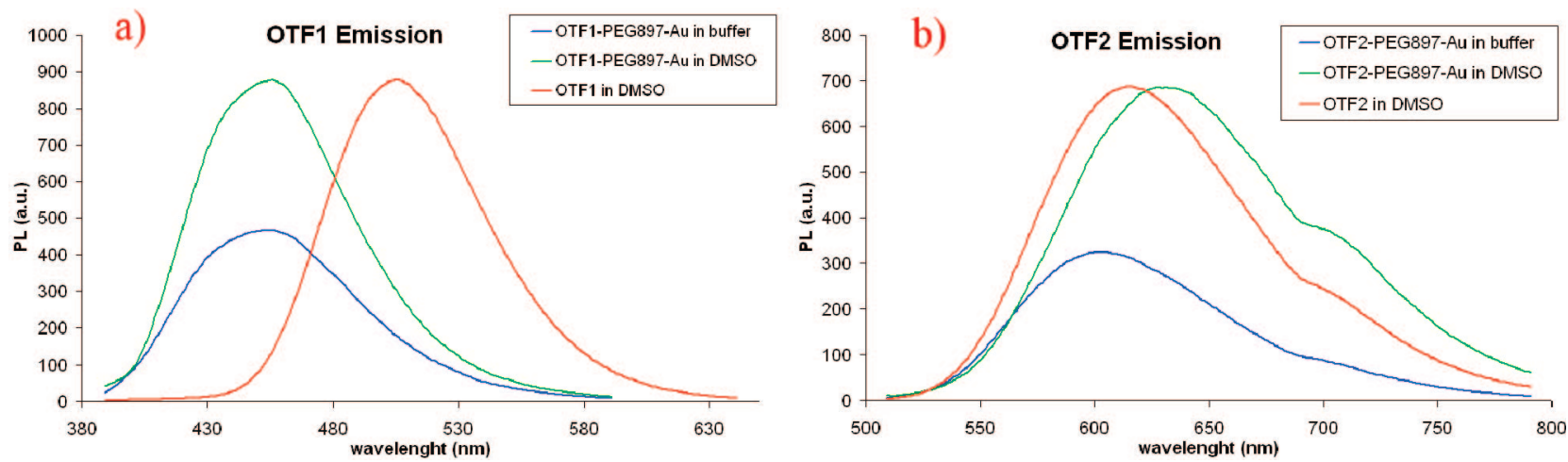

Figure 3. Comparison of emission spectra of OTF1-Au (a) and OTF2-Au (b) dissolved in both DMSO and aqueous buffer with respect to that of the free OTF molecules in DMSO. While for the OTF1-Au there was no shift of the conjugates when the buffer was exchanged from water to DMSO, in the case of OTF2-Au conjugates a red shift of $30 \mathrm{~nm}$ was observed. In both cases however when exchanging the solvent from water to DMSO an increase in the PL signal was observed (the spectra were recorded at the same Au nanoparticle concentration).

Table 2. Average Hydrodynamic Diameters (as determined via DLS) of Polymer-Coated NPs, Diamino-PEG-Functionalized NPs, and OTF-NP Conjugates, for Samples Characterized by Different Types of Inorganic Cores (Either Au or $\Gamma-\mathrm{Fe}_{2} \mathrm{O}_{3}$ ), and Molecular Weights of the Diamino-PEG Molecules ${ }^{a}$

\begin{tabular}{lc}
\hline \multicolumn{1}{c}{ sample name } & size $(\mathrm{nm})$ \\
\hline$\gamma$-Fe $\mathrm{O}_{3}$-PC & $17.9 \pm 0.3$ \\
$\gamma$-Fe $\mathrm{F}_{3}$-PEG3400 & $24.1 \pm 0.5$ \\
$\gamma$-Fe $2 \mathrm{O}_{3}$-PEG3400-OTF2 & $26.9 \pm 1.8$ \\
$\mathrm{Au}-\mathrm{PC}$ & $10.5 \pm 1.8$ \\
$\mathrm{Au}-\mathrm{PEG} 2000$ & $19.5 \pm 1.4$ \\
$\mathrm{Au}-\mathrm{PEG} 2000-$ OTF2 & $21.0 \pm 1.7$ \\
$\mathrm{Au}-\mathrm{PEG} 3000$ & $21.4 \pm 1.2$ \\
$\mathrm{Au}-\mathrm{PEG} 3000-$ OTF2 & $23.1 \pm 1.9$ \\
$\mathrm{Au}-\mathrm{PEG} 3400$ & $20.6 \pm 1.3$ \\
$\mathrm{Au}-\mathrm{PEG} 3400-$ OTF2 & $23.3 \pm 1.8$ \\
Au-PEG6000 & $29.5 \pm 1.9$ \\
Au-PEG6000- OTF2 & $32.7 \pm 1.9$
\end{tabular}

${ }^{a}$ By increasing the molecular weight (i.e., chain length) of the diamino-PEG molecules attached to the NPs, larger diameters were measured, while almost the same increase in the hydrodynamic diameter (between 1.5 and $2.8 \mathrm{~nm}$ ) for all types of diamino-PEG-functionalized NPs was recorded when OTF molecules were anchored to them.

related straightforwardly to the length of the diamino-PEG molecules employed (hence to their molecular weight, see Table 2).

In the following step of OTF functionalization, binding of the same OTF to the NPs functionalized with PEG molecules having different molecular weights led, on the other hand, to a constant increase in diameter, which corresponded to an increase in size between 1.5 and $2.8 \mathrm{~nm}$, and this did not depend on the type of diamino-PEG-functionalized NPs.

3.4. Stability of the Conjugates and Magnetic Response. TEM images of the OTF-NP conjugates showed no remarkable signs of aggregation on the carbon support film of the TEM grids. In addition, no noticeable differences in the type of patterns formed on the film either on average interparticle distance could be observed when comparing TEM images of NPs before and after conjugation to OTFs, which confirmed that the conjugates are based on individual NPs and that no major structural or aggregation effects take place as a result of the conjugation reaction. (Figure 1s, SI). Also, salt and $\mathrm{pH}$ stability tests led us to conclude that the $\mathrm{OTF}-\mathrm{NP}$ conjugates do not precipitate under physiological conditions $(\mathrm{pH} 7$ and $114 \mathrm{mM} \mathrm{NaCl}$ ) and that they are stable in a $\mathrm{pH}$ range from basic $(\mathrm{pH} 9)$ to acidic ( $\mathrm{pH}$ 3) conditions.

The fluorescence stability of the conjugates, on the other hand, was assessed via comparison with that of fluorescein-based NPs conjugates that were prepared by reacting diamino-PEGfunctionalized NPs with NHS-fluorescein (data not shown). We could observe that while fluorescein-NP conjugates underwent a complete loss of fluorescence already a few days after their preparation, the OTF-NPs conjugates, specifically those based on the OTF1 and OTF2 samples, could be stored for several months without undergoing any degradation of the fluorescent properties.

Besides the oligothiophene OTF1, emitting in the blue, and OTF2, emitting in the orange, which were thoroughly investigated and shown to be stable dye molecules for preparation of such hybrid fluorescent magnetic conjugates and gold-based conjugates, we also tested the conjugation of NPs with other OTF molecules. Through these experiments we could conclude that we were able to link successfully most of the OTF molecules to the NPs (see Table 1s, SI).

In a few cases the conjugation reaction between diaminoPEG-functionalized NPs and the OTF molecules did not occur, as formation of the conjugates could not be confirmed by gel electrophoresis. In these cases, the fluorescence signal from the OTF could be detected only in the loading well. This occurred, for instance, with the OTF sample named OTF8 (see Figure 4s, $\mathrm{SI}$ ), for which it is likely that the poor availability of the NHS group and its molecular structure render this specific OTF molecule scarcely accessible to the nanoparticle and also poorly soluble in water. We believe that these were critical parameters for the failure of the linking reaction. In other cases although the conjugation reaction to the NPs was successful, as confirmed by colocalization of the bands on the agarose gel, a loss of fluorescence of the conjugates was observed if they were exposed to the UV lamp for a time longer than $10 \mathrm{~min}$ (see Table 1s, SI, for the case of OTF4- and OTF5-based conjugates). In these cases we concluded that the stability of the OTF-NP conjugates was related to the structure of the specific OTF under study and the conformation that it took once linked to the NPs. In the case of OTF4 and OTF5, for instance, we can speculate that the binding of these molecules to the NPs modifies their configuration such that they are now characterized by a lower degree of p-electron conjugation (i.e., lower fluorescence) and that presumably this change adversely affects their photostability.

In the case of the OTF $-\gamma-\mathrm{Fe}_{2} \mathrm{O}_{3}$ conjugates, the magnetic response was macroscopically observed by placing a small magnet close to a vial containing the solution of conjugates. A 


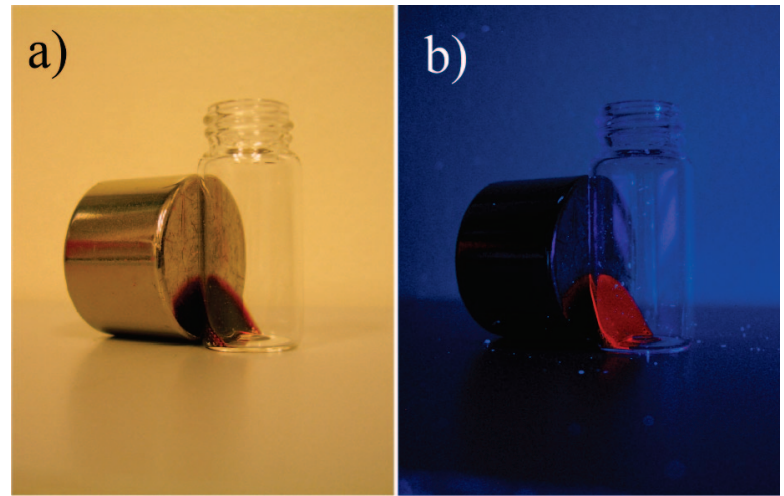

Figure 4. Magnetic response of OTF2-iron oxide conjugates. A solution of conjugates was promptly attracted to the $\mathrm{NdFeB}$ magnet placed close to the vial. The images of the conjugates were taken under visible light (a), which evidenced the adsorption color of iron oxide NPs, and under UV light exposure (b), by which the fluorescence of the OTFs could be seen.

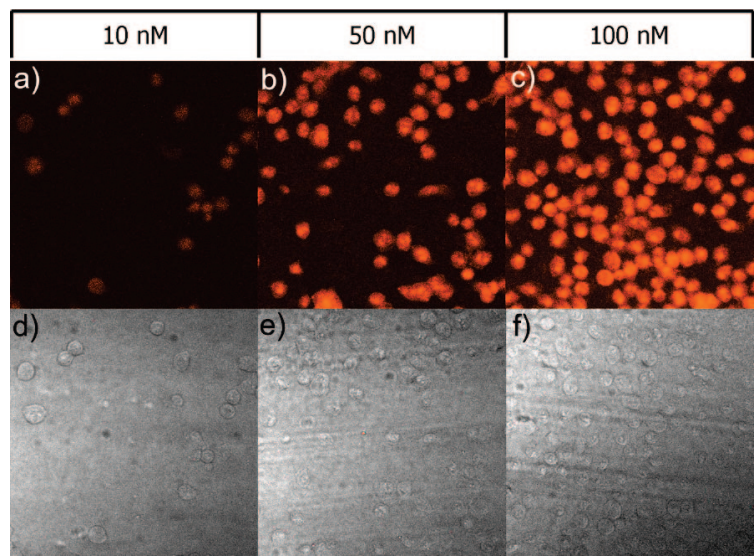

Figure 5. Confocal microscopy images of KB cells doped with OTF2-iron oxide conjugates at different concentrations: (a and d) $10 \mathrm{nM}$, (b and e) 50 $\mathrm{nM}$, and (c and f) $100 \mathrm{nM}$. $(\mathrm{a}-\mathrm{c})$ Acquired under excitation by the $488 \mathrm{~nm}$ line of an argon laser using a $605 \pm 15 \mathrm{~nm}$ filter. (d-f) Acquired under bright field exposure.

sudden noticeable attraction of the conjugates to the lateral wall of the vial, where the magnet was positioned, could be observed (Figure 4).

As a general remark about the protocol that we developed to attach OTFs to NP we should point out that although our procedure involved different reaction steps and different purification steps and time lapses required per each step, the chemical and physical stability of the resulting conjugates is high (as confirmed by optical absorption and emission spectra, gel electrophoresis runs, TEM analysis, and $\mathrm{pH}$ and salt stability, which were run periodically on stored samples).

3.5. Cell Studies Based on OTF-NP Conjugates: Cell Uptaking, Fluorescent and Magnetic Behavior, and Biocompatibility. Figure 5 reports representative images of cell samples to which solutions of OTF2 $-\gamma-\mathrm{Fe}_{2} \mathrm{O}_{3}$ conjugates (at concentrations of conjugates equal to 10,50 , and $100 \mathrm{nM}$ ) had been administered. The concentration of the conjugates added to the culture media was referred to that of the NPs. The set up parameters used for imaging the three samples were identical. Figure 5a, 5b, and 5c shows the fluorescent signals detected at $605 \mathrm{~nm}$ emission due to the OTF2, while Figure 5d, 5e, and $5 \mathrm{f}$ shows the corresponding bright field images. From a qualitative point of view, we could appreciate a difference in the intensity of fluorescence when comparing the results of the three experiments carried out at different concentrations of conjugates. This gave us an indication of the increasing nonspecific uptaking by the cells. Furthermore, in all these experiments the cells preserved a normal morphology and no alteration of the cell functions was recorded. Also, the fluorescence images showed that already at a conjugate concentration of $10 \mathrm{nM}$ the fluorescence signal was strong enough within the doped cells (Figure 5). A $z$-scan study was performed in order to localize the conjugates within cells (Figure 6). By focusing on the lower plane of the cells that were adherent to the substrate and moving up along the $z$ axis with a step size of $2 \mu \mathrm{m}$, we could capture the fluorescent signal distribution under excitation by blue light. An intense fluorescence was localized just at the middle height of the cells, where the cytoplasm is located. This clearly indicated an intracellular storage of the conjugates within the cell and not at their membrane surface.

Besides the maintenance of the fluorescent signal within the cells (once the conjugates had been uptaken by the cells) it was important to evaluate the cytotoxicity effect of the conjugates. To this aim we used the standard MTT test, as described in the Experimental Section. ${ }^{72}$ This was carried out on the individual components that formed the conjugate (i.e., both the diaminoPEG-functionalized NPs and the free NHS-OTFs transferred in water) and on the final OTF-NP conjugates as a function of the concentration administered to the culture medium. The results are reported in Figure 7. The trends show clearly that in all cases studied the cell mortality rate increased as a function of the concentration added to the media both for the individual components and for the conjugates. Also, at concentrations higher than $50 \mathrm{nM}$ the toxicity of the resulting conjugates was more pronounced than that of the individual components, therefore indicating that the presence within the same nanostructure of both the NPs and the OTFs leads to a synergetic toxic effect on cells.

The increased toxicity of the conjugates with respect to the bare components was less pronounced for $\gamma-\mathrm{Fe}_{2} \mathrm{O}_{3}$-based conjugates than for gold-based ones. In general, a reduced cytotoxicity of the OTF $-\gamma-\mathrm{Fe}_{2} \mathrm{O}_{3}$ conjugates was observed (with a mortality rate lower than 20\%) when the concentration of the administered conjugates was lower than $100 \mathrm{nM}$. It is not straightforward to compare the toxicity of our conjugates with that of other iron oxide- or gold-based nanostructures reported so far in the literature. This is mainly because several parameters, including the size of the NPs, the specific type of NP coating, the type of cells studied, and so on, can have a strong effect on the overall cytotoxicity. We can say however that the toxicity of our particles is on the same order of magnitude as that found in several relevant works in the field, ${ }^{73-75}$ although the NP size and coating as well as the types of cells studied in those reports differed from the ones examined in the present work. However, the cytotoxicity found for our conjugates does not prevent application of the OTF-NP nanostructures for cellular studies.

It is worth noting that the concentration for the OTF-NP conjugates was referred to the NPs concentration, while for the free OTF we considered a concentration range 20 times higher than that of the NPs. This estimate was based on previous

(73) Thomas, M.; Klibanov, A. M. Proc. Natl. Acad. Sci. U.S.A. 2003, 100 (16), 9138-9143.

(74) Samanta, B.; Yan, H.; Fischer, N. O.; Shi, J.; Jerry, D. J.; Rotello, V. M. J. Mater. Chem. 2008, 18 (11), 1204-1208.

(75) Simioni, A. R.; Primo, F. L.; Rodrigues, M. M. A.; Lacava, Z. G. M.; Morais, P. C.; Tedesco, A. C. IEEE Trans. Magn. 2007, 43 (6), 24592461. 


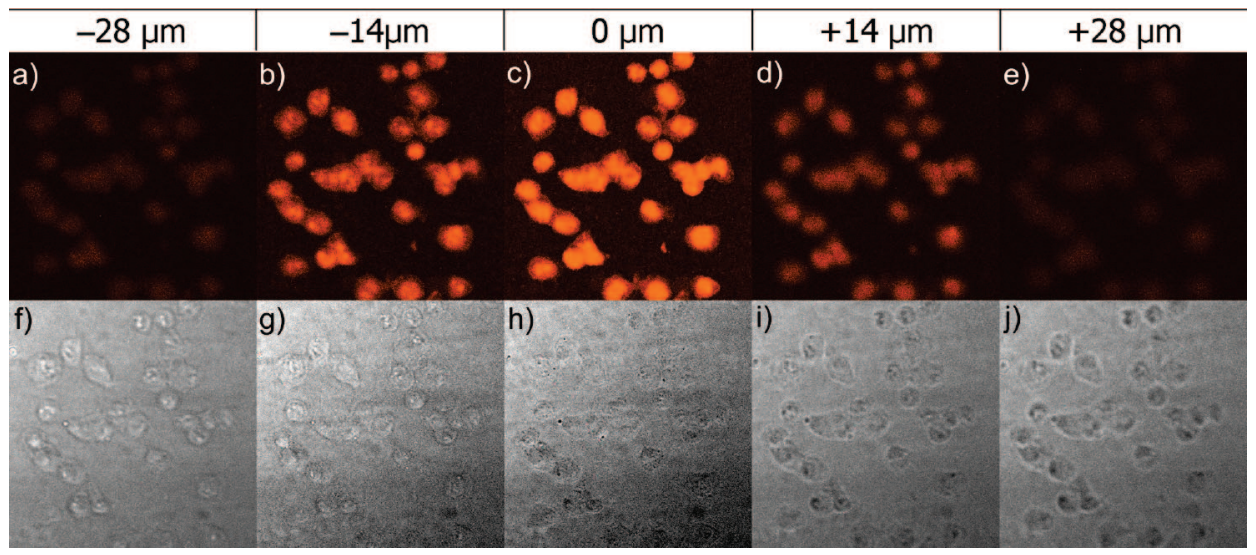

Figure 6. Confocal microscope $z$-scan sections of KB cells doped with $50 \mathrm{nM} \mathrm{OTF2-iron} \mathrm{oxide} \mathrm{conjugates.} \mathrm{The} \mathrm{images} \mathrm{were} \mathrm{acquired} \mathrm{with} \mathrm{a} \mathrm{step} \mathrm{size} \mathrm{of}$ $2 \mu \mathrm{m}$. Here, from left to right (panels a-e and $\mathrm{f}-\mathrm{j}$ ), we only show images of sections separated from each other by a step size of $14 \mu \mathrm{m}$. The images of panels a-e were acquired using the $488 \mathrm{~nm}$ line of an argon laser and a $605 \pm 15 \mathrm{~nm}$ filter, whereas the images of panels $\mathrm{f}-\mathrm{j}$ were acquired under bright field exposure.


Figure 7. Cytotoxicity assay of gold- (a) and iron oxide- (b) based conjugates on KB cells. The viability trends (via the MTT assay) were obtained by administering different concentrations of individual components (i.e., either PEG-functionalized NPs or OTFs in water) and OTF-NP conjugates. Administered concentrations were between 1 and $200 \mathrm{nM}$ for both NPs and OTF2-NP conjugates and between 20 and $4000 \mathrm{nM}$ for the free OTF2 in water.

experimental evidence ${ }^{70}$ according to which the number of PEG molecules attached per NP under the conditions described in the Experimental Section is between 15 and 20, and thus, an equivalent number of NHS-OTF molecules is expected to be linked to the NP surface (as we performed the conjugation reaction in the presence of a large excess of OTF molecules per NP).

To demonstrate the application of OTF-based conjugates in multiplexing labeling of cells we mixed together the samples of $\mathrm{KB}$ cells doped, respectively, with $\mathrm{OTF} 1-\gamma-\mathrm{Fe}_{2} \mathrm{O}_{3}$ and $\mathrm{OTF} 2-\gamma-\mathrm{Fe}_{2} \mathrm{O}_{3}$ and imaged them under a confocal microscope.

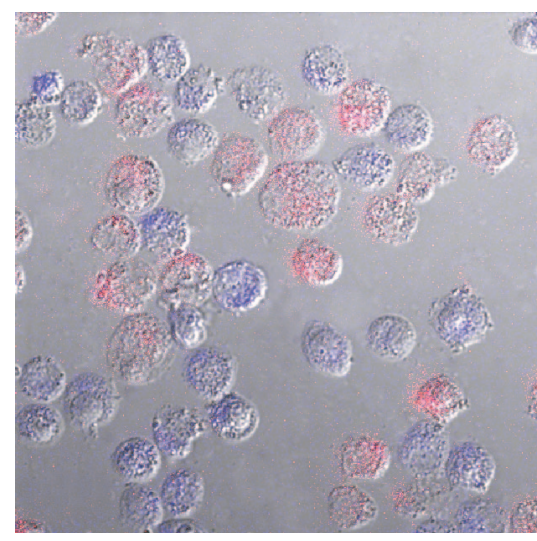

Figure 8. Typical confocal image of $\mathrm{KB}$ cells doped, respectively, with $\mathrm{OTF} 1-\gamma-\mathrm{Fe}_{2} \mathrm{O}_{3}$ (blue-emitting cells) and $\mathrm{OTF} 2-\gamma-\mathrm{Fe}_{2} \mathrm{O}_{3}$ conjugates (orange emitting cells). The image was obtained by merging the phase contrast image and the fluorescent image (the latter was taken with acquisition channels at $450 \pm 20 \mathrm{~nm}$ for OTF1 $-\gamma-\mathrm{Fe}_{2} \mathrm{O}_{3}$ and $620 \pm 20$ $\mathrm{nm}$ for OTF2 $-\gamma-\mathrm{Fe}_{2} \mathrm{O}_{3}$, respectively, and using a $405 \mathrm{~nm}$ excitation laser).

We used the same wavelength of excitation, which corresponded to $405 \mathrm{~nm}$, and we acquired the fluorescent signals of the blueemitting OTF $1-\gamma-\mathrm{Fe}_{2} \mathrm{O}_{3}$ conjugate in the $450 \pm 20 \mathrm{~nm}$ spectral range and the orange-emitting OTF2 $-\gamma-\mathrm{Fe}_{2} \mathrm{O}_{3}$ conjugate in the $620 \pm 20 \mathrm{~nm}$ spectral range. As observed in Figure 8, it is possible to image both the orange and blue fluorescent cells using a single excitation source, which clearly represents an advantage of our OTF-NP conjugates in multiplexing experiments with respect to standard organic dyes.

The study of the magnetic response of $\mathrm{KB}$ cells doped with $\mathrm{OTF}-\gamma-\mathrm{Fe}_{2} \mathrm{O}_{3}$ was carried out by culturing the doped $\mathrm{KB}$ cells in a Petri dish under which a small magnet had been placed followed by exchange with fresh buffer, as described in the Experimental Section. Typical images of the adherent cells in the region above the magnet and far from this zone are shown in Figure 9. We observed, both in contrast phase and in fluorescence mode, that there were more adherent cells in the region underneath which the magnet had been placed than far from this region. This indicated clearly that the doped cells had been attracted by the magnet. It is worth noting that we used an adherent cell lineage as we wanted to look only at adhering cells cultured in the presence of the magnet, and therefore, the statistical study was carried out only on those cells. Indeed, the 

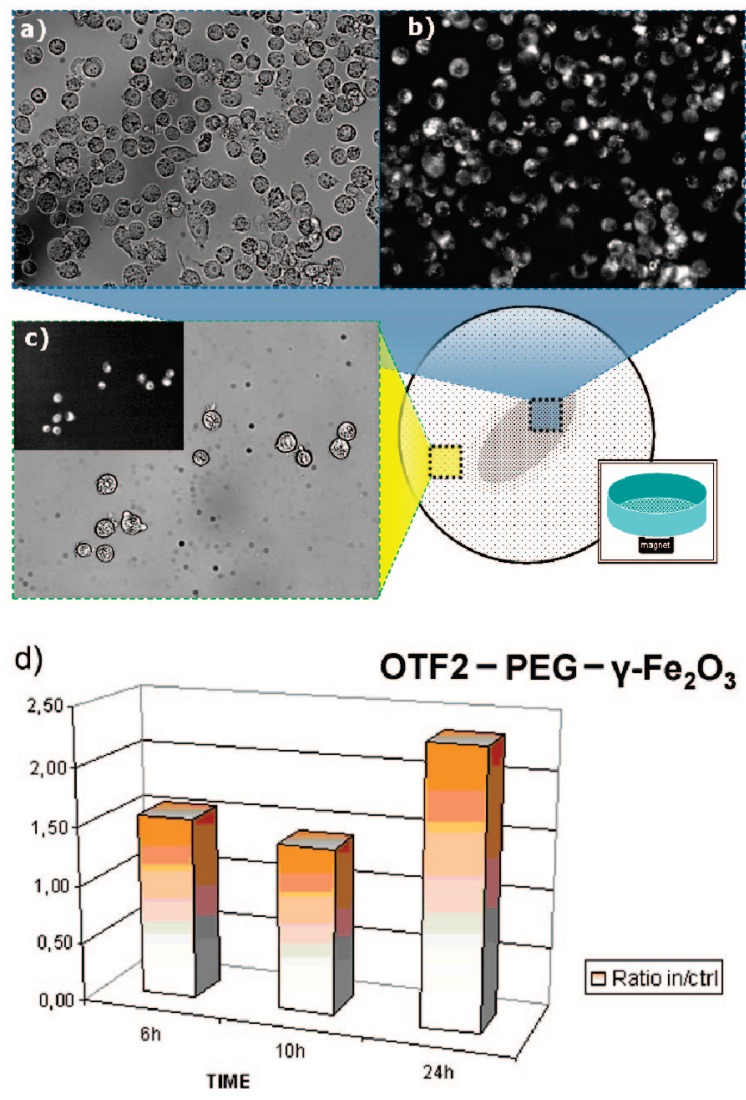

Figure 9. Magnetic assay on $\mathrm{KB}$ cells doped with $\mathrm{OTF} 2-\gamma-\mathrm{Fe}_{2} \mathrm{O}_{3}$ conjugates. Doped KB cells were cultured in the presence of a small magnet placed underneath a Petri dish. After an incubation time of $24 \mathrm{~h}$ at $37{ }^{\circ} \mathrm{C}$ and after the medium had been exchanged with fresh medium to remove the cells in suspension, images were taken both in the region underneath which the magnet had been placed $(a$ and $b)$ and far $(1.5 \mathrm{~cm})$ from this region (c and inset). Images a and $\mathrm{c}$ were taken under bright field while image $b$ and inset of image $c$ were taken under UV exposure.(d) Statistical analysis of doped $\mathrm{KB}$ cells attracted by the magnet versus the incubation time. The graph describes the ratio between the cells counted in the region where the magnet was placed with respect to a control sample (doped cells seeded at the same density but in the absence of the magnet) as a function of the incubation time.

number of total cells that could be imaged on top of the magnet before removing the cells left in suspension (i.e., before exchanging the media) was much higher than that after exchange with fresh medium (see, for instance, Figure 9s, SI) since in the latter case only the cells adhering to the substrate remained and could be imaged. Although in fact the magnet attracts a large number of cells, not all of them find a place to adhere on the substrate once the first layer of cells has coated the surface area available on the Petri dish. As observed both in bright field and in fluorescent mode, several clusters of cells are visible on a plane higher than the plane of the adhering cells (Figure 9s, SI).

The number of adhering cells in the region above the magnet was compared to that of control samples in which doped cells had been cultured under the same conditions except that no magnet was placed underneath the dish. We report in Figure $9 \mathrm{c}$ the ratio of cells entrapped in the region on top of the magnet with respect to that of the control sample for $\mathrm{KB}$ cells doped with OTF2 $-\gamma-\mathrm{Fe}_{2} \mathrm{O}_{3}$ conjugates (with PEG897 as PEG molecules attached to the nanoparticle surface) as a function of the incubation time. We observed that the extent of attraction was related to the incubation time as the maximum effect of the magnet attraction was observed over a longer incubation time. After $24 \mathrm{~h}$ of incubation the ratio of adherent cells in the area on top of the magnet to those of the control experiment was around 2.5.

A similar behavior was also observed for the other OTF-NP conjugates (data not shown). While this enrichment factor might seem low, we should point out that we counted only adherent cells and discarded all those cells that during incubation had moved in the area close to the magnet but could not adhere to the substrate and remained instead in solution (and as such were washed away, see Figure 9s, SI). The cell response to the magnet however took some hours, and this is due to several reasons. First, our test was based on single colloidal iron oxide NPs with a diameter equal to $8 \mathrm{~nm}$, which therefore have a small magnetic moment, and in addition the field generated by the magnet was only equal to $1.3 \mathrm{~T}$. Furthermore, it is clear that the tumor cells are preferentially accumulated close to the magnet only if they uptake the conjugates. In this respect, the influence of PEG molecules, which reduce the nonspecific uptaking by the cells, should be responsible for the slow response to the magnet. A study is in progress in our group on the quantification of the intracellular amount of conjugates engulfed by the cells as a function of the PEG molecules used for preparing the OTF-NP conjugates. We should point out, on the other hand, that reduced nonspecific uptaking will be desirable whenever specific targeting experiments will be carried out.

\section{Conclusions}

In summary, we presented a rational design for the preparation of OTF-NPs conjugates mediated by diamino-PEG molecules that act as bridging molecules. In the reaction scheme developed to prepare such conjugates (Figure 1a) we could vary independently the type of inorganic NPs (we reported here results for both gold and iron oxide NPs, but the scheme should be extendable to other types of NPs easily), the molecular weight of the PEG molecules (therefore the surface properties of the conjugates), and the type of OTF molecules as well (hence, the emission color of the conjugates).

The stability of the linkage between NPs and OTFs was established by several techniques, such as gel electrophoresis, PL measurements, and dynamic light scattering. Furthermore, the average hydrodynamic diameters of the conjugates, always below $33 \mathrm{~nm}$, along with TEM characterization indicated a substantial absence of aggregation. The $\mathrm{pH}$ and salt tests allowed us to verify the stability of the conjugates under physiological conditions and suggest the suitability of such fluorescent-magnetic conjugates for in vivo applications. For this purpose in fact longer blood circulation times are required, and these are strongly related to the size of the specific nano-object, its monodispersity, and its physiological stability. In addition, one important advantage of the present OTF-NP conjugates is that they can be prepared well before their intended use as they can be stored for several months without compromising their fluorescent and magnetic properties.

In this study we focused mainly on the interaction of magnetic-fluorescent conjugates based on $\mathrm{OTF}-\gamma-\mathrm{Fe}_{2} \mathrm{O}_{3}$ conjugates with tumor cells and also on their response to an external magnetic field. Once taken up by the tumor cells, the conjugates have been shown to be promising tools for combined optical detection and magnetic separation/delivery experiments. The fluorescence signal was clearly detectable even when the concentration of the conjugates in the solution that was administered to the cells was such that the mortality of the cells 
was less than $20 \%$, at least for the iron-based conjugates (with an administered concentration below $100 \mathrm{nM}$ ).

In addition, by performing a multilabeling experiment we could demonstrate the suitability of OTFs - NP conjugates in multiplexing experiments in which just one single excitation wavelength is required. Finally, the possibility to reduce the specific uptaking of these conjugates by choosing the right PEG molecules makes them appealing in a future study for the receptor surface functionalization and specific targeting and testing them in in vivo systems on animal models. Experiments are currently under way in our group in this direction.

Supporting Information Available: TEM images of the bare NPs and conjugates, further gel characterization including some control experiments, gel extraction and dialysis procedure, absorption and emission spectra of the conjugates, and fluo- rescent and phase contrast images of the magnetic assay; absorption, emission, and FT-IR spectra and gel characterization of free OTFs are also included. This material is available free of charge via the Internet at http://pubs.acs.org.

Acknowledgment. Thanks are due to Mediteknology srl for supplying the oligothiophene fluorophores used in this study and Massimo Zambianchi and Laura Favaretto (ISOF-CNR, Bologna) for helpful assistance. We thank also Davide P. Cozzoli, Benedetta Antonazzo, and Raffaella Buonsanti for providing the iron oxide nanoparticles. This work was supported in part by the Italian Ministry of Research under FIRB Grants RBN01KJHT-007, RBIN048TSE, and RBLA03ER38 and in part by the European STREP project SA-NANO under Contract 013698.

JA800102V 